\title{
UNIFORMLY CONTINUOUS SOLUTIONS OF DIFFERENCE EQUATIONS WITH CONTINUOUS TIME
}

\author{
YU. L. MAISTRENKO \\ Institute of Mathematics, Academy of Sciences of the Ukrainian S.S.R. \\ Kiev, U.S.S.R.
}

For nonlinear difference equations with continuous argument (time),

$$
x(t+1)=f(x(t)), \quad t \in R^{+},
$$

typical are so-called asymptotically discontinuous solutions - continuous and bounded but not uniformly continuous on the semiaxis (see [1] and [2]). It is interesting to study those in some sense exceptional situations, when asymptotically discontinuous solutions of the equation (1) do not exist; i.e., when every solution $R^{+} \ni t \rightarrow x(t)$ is uniformly continuous.

For equation (1) we pose an initial value problem

$$
x(t)=\varphi(t), \quad t \in[0,1]
$$

and assume that

$$
f \in C(I, I), \quad \varphi \in C([0,1], I),
$$

where $I \subset R$ is some closed bounded interval. Moreover, let us assume that the condition of compatibility is fulfilled:

$$
\varphi(1)=f(\varphi(0)) \text {. }
$$

It is easy to see that the solution $x(\cdot)$ of (1) and (2) exists, is unique and can be written as

$$
x(t)=f^{n}(\varphi(t-n)), \quad t \in[n, n+1], n \in Z^{+},
$$

where $f^{n}$ is the $n$th iterate of $f$. Therefore, in view of (3) and (4)

$$
x(\cdot) \in C\left(R^{+}, I\right) .
$$

From now on we assume that conditions (3) and (4) are fulfilled and thus solutions satisfying (6) are only considered. The set of fixed points and the set of periodic points of $f$ will be denoted by $\operatorname{Fix}(f)$ and $\operatorname{Per}(f)$, respectively. 
THEOREM. The following conditions are equivalent:

(i) all solutions of equation (1) are uniformly continuous,

(ii) the family $\left\{f^{n}\right\}_{n \in Z^{+}}+$is equicontinuous,

(iii) the set $\operatorname{Per}(f)$ is connected and equals either $\operatorname{Fix}(f)$ or $\operatorname{Fix}\left(f^{2}\right)$,

(iv) there exists an interval $\left[x_{1}, x_{2}\right] \subset I \stackrel{\text { def }}{=}[a, b], x_{1} \leqslant x_{2}$ such that

$$
\begin{array}{cl}
g(x)=x & \text { for } x \in\left[x_{1}, x_{2}\right], \\
g(x)>x & \text { for } x \in\left[a, x_{1}\right], \\
g(x)<x & \text { for } x \in\left(x_{2}, b\right), \\
g^{2}(x) \neq x & \text { for } x \in I \backslash\left[x_{1}, x_{2}\right],
\end{array}
$$

where $g=f$ or $g=f^{2}$.

Proof (i) $\Rightarrow$ (ii). Assume that the family $\left\{f^{n}\right\}_{n \in Z^{+}}$is not equicontinuous. We will show that in this case equation (1) has asymptoticlly discontinuous solutions. Since $\left\{f^{n}\right\}_{n \in Z_{+}}$is not equicontinuous one can find a sequence $\left\{V_{i}\right\}_{i \in Z^{+}}$of nested intervals $V_{i} \supset V_{i+1}$ for $i \in Z^{+}$such that $\bigcap_{i \geqslant 0} V_{i}=\left\{x^{*}\right\}$, and an increasing sequence $\left(n_{i}\right)$ of positive integers such that $\operatorname{diam}\left(f^{n_{i}}\left(V_{i}\right)\right) \varepsilon$ for all $i \in Z^{+}$and some $\varepsilon>0$.

Let $x(\cdot)$ be the solution with an initial function $\varphi$. We choose $\varphi$ in such a way that the set of its values (a closed interval, in view of continuity of $\varphi$ ) contains $x^{*}$ in its interior. Moreover, we assume that the graph of $\varphi$ intersects the line $x=x^{*}$ at least in one point $t^{*} \in(0,1)$ i.e. for any $\delta>0$ the set $\varphi\left(U_{\delta}\left(t^{x}\right)\right)$ contains a whole neighbourhood of $x^{*}$. (By $U_{\delta}(B)$ we denote the $\delta$ - neighbourhood of $B$.) Any such solution $x(\cdot)$ is not uniformly continuous on the semiaxis. Indeed $\operatorname{diam}\left(x\left(U_{i}\right)\right)>\varepsilon$ for all $i \in Z^{+}$, where $U_{i}$ $=\left\{t \in\left[n_{i}, n_{i+1}\right], t=n_{i} \in V_{i}\right\}$, but $\operatorname{diam}\left(U_{i}\right)=\operatorname{diam}\left(V_{i}\right) \rightarrow 0$ as $i \rightarrow \infty$

(ii) $\Rightarrow$ (i). The implication is a simple consequence of the equicontinuity of $\left\{f^{n}\right\}_{n \in Z^{+}}$, the uniform continuity of $\varphi$, and formula (5).

(ii) $\Rightarrow$ (iii). First we show that the equicontinuity of $\left\{f^{n}\right\}_{n \in Z^{+}}$implies connectedness of Fix $(f)$. Suppose the contrary. Since $f$ is continuous, we can find two points $x^{\prime}, x^{\prime \prime} \in \operatorname{Fix}(f)$ such that $\left(x^{\prime}, x^{\prime \prime}\right) \cap \operatorname{Fix}(f)=\emptyset$. Let for definitness $f(x)>x$ for $x \in\left(x^{\prime}, x^{\prime \prime}\right)$. Then $\sup f^{n}(x) \geqslant x^{\prime \prime}$ for $x \in\left(x^{\prime}, x^{\prime \prime}\right)$ and $f^{n}\left(x^{\prime}\right)$ $=x^{\prime}$ for any $n \in Z^{+}$. Therefore, for any small semineighbourhood $\left[x^{\prime}, x^{\prime}+\delta\right)$ of the point $x$ we have

$$
\sup _{n} \operatorname{diam}\left(f^{n}\left(\left[x^{\prime}, x^{\prime}+\delta\right)\right)\right) \geqslant\left|x^{\prime}-x^{\prime \prime}\right|
$$

which contradicts the equicontinuity of $\left\{f^{n}\right\}_{n \in Z^{+}}$. Consequently the set Fix $(f)$ is connected. Analogously one can prove that the sets $\operatorname{Fix}\left(f^{\prime \prime}\right), n \in Z^{+}$ are connected, and therefore the set $\operatorname{Per}(f)$ is connected. The fact that $\operatorname{Per}(f)$ 
in this case equals either $\operatorname{Fix}(f)$ or $\operatorname{Fix}\left(f^{2}\right)$ follows from the following. Assume that $f$ has a cycle $\left\{x_{1}, \ldots, x_{m}\right\}$ of some period $m>2$, where $x_{i}$ $<x_{i+1}$ for $i=1, \ldots, m-1$. Then

$$
f^{m}(x)=x \quad \text { for all } x \in\left[x_{1}, x_{m}\right]
$$

It is well known that for a continuous function this implies that the equality (8) fulfilled for $m=1$ or $m=2$. Thus the map $f$ can have only cycles of period either 1 or 2 .

The conditions (iv) are a simple decoding of the conditions (iii) and therefore we omit the proof of their equivalence. Hence, in order to complete the proof of the theorem it is sufficient to show that (iv) implies (ii).

(iv) $\Rightarrow$ (ii). Let the inequalities (7) be fullfiled for $g=f$. We shall show that then the sequence $\left(f^{n}\right)$ is uniformly convergent to some function and this will quarantee the equicontinuity of $\left\{f^{n}\right\}_{n \in Z^{+}}$. By (7) we have $\operatorname{Per}(f)$ $=\operatorname{Fix}(f)=\left[x_{1}, x_{2}\right]$, hence the trajectory $\left\{f^{n}(x)\right\}_{n \in Z^{+}}$of any point $x \in I$ is attracted by the interval $\left[x_{1}, x_{2}\right]$. Moreover if this trajectory hits the interior of $\left[x_{1}, x_{2}\right]$ in some time, it stays there. We split $I$ into two subsets $X_{1}$ and $X_{2}$, where

$$
X_{1}^{\cdot}=\left\{x \in I: \exists n_{0}=n_{0}(x), f^{n_{0}}(x) \in\left[x_{1}, x_{2}\right]\right\}, \quad X_{2}=I \backslash X_{1} .
$$

The points of $X_{2}$ are attracted under $f$ to one of the endpoints of $\left[x_{1}, x_{2}\right]$. But, in general before falling into the domain of the immediate attraction of $x_{1}$ or $x_{2}$, the trajectory $\left\{f^{n}(x)\right\}_{n \in Z^{+}}\left(x \in X_{2}\right)$ can go around the interval $\left[x_{1}, x_{2}\right]$ a few times. Nevertheless, there exists $n_{1}$ such that for all $x \in X_{2}$ and $n>n_{1} f^{n}(x)$ lies in the domain of the immediate.attraction of either $x_{1}$ or $x_{2}$ and thus is monotonically attracted to one of these points. Therefore

$$
X_{2}=X_{2,1} \cup X_{2,2} \text {, }
$$

where

$$
\begin{aligned}
& X_{2,1}=\left\{x \in X_{2}: f^{n}(x) \rightarrow x_{1} \text { as } n \rightarrow \infty\right\}, \\
& X_{2,2}=\left\{x \in X_{2}: f^{n}(x) \rightarrow x_{2} \text { as } n \rightarrow \infty\right\} .
\end{aligned}
$$

It is also clear that $X_{2,1} \cap X_{2,2}=\emptyset$. We define the limit function $f_{0}$ by

$$
f_{0}(x)= \begin{cases}f^{n_{0}}(x) & \text { if } x \in X_{1}, \\ x_{1} & \text { if } x \in X_{2,1}, \\ x_{2} & \text { if } x \in X_{2,2}\end{cases}
$$

The uniform convergence of the sequence $\left(f^{n}\right)$ to $f_{0}$ is a consequence of the described properties. In the case when inequalities (7) hold for $g=f^{2}$, the proof is analogous, with the difference that the sequence $\left(f^{n}\right)$ has two cluster 
points

$$
\begin{gathered}
\lim _{n \rightarrow \infty} f^{2 n}(x)=\left(f^{2}\right)_{0}(x), \\
\lim _{n \rightarrow \infty} f^{2 n+1}(x)=f\left(\left(f^{2}\right)_{0}(x)\right),
\end{gathered}
$$

where $\left(f^{2}\right)_{0}$ is defined by (9). Thus the theorem is proved.

Corollary. If the inequalities (7) are fulfilled for $g=f$, then

$$
\lim _{n \rightarrow \infty} x(t+n)=(f)_{0}(\varphi(t))
$$

uniformly in $t \in[0,1]$.

If the inequalities (7) are fulfilled for $g=f^{2}$, then

$$
\begin{gathered}
\lim _{n \rightarrow \infty} x(t+2 n)=\left(f^{2}\right)_{0}(\varphi(t)), \\
\lim _{n \rightarrow \infty} x(t+2 n+1)=f\left(\left(f^{2}\right)_{0}(\varphi(t))\right),
\end{gathered}
$$

uniformly in $t \in[0,1]$.

\section{References}

[1] A. N. Sharkovskii and E Yu. Romanenko, Asymptotic behaviour of solutions of differential-difference equations, in: Qualitive methods for studying nonlinear differential equations and nonlinear oscillations, Kiev, Inst. of Math. AN USSR, 1981, 171-199, in Russian.

[2] A. N. Sharkovskii, Yu. Maistrenko, Turbulence and simple hyperbolic systems, in: Mathematical Analysis, Teubnertexte zur Mathematik, Band 79, Leipzig 1985, 220-235. 
DYNAMICAL SYSTEMS AND ERGODIC THEORY BANACH CENTER PUBLICATIONS, VOLUME 23 PWN - POLISH SCIENTIFIC PUBLISHERS

WARSZAWA 1989

\title{
HOW COMPLICATED CAN BE ONE DIMENSIONAL DYNAMICAL SYSTEMS: DESCRIPTIVE ESTIMATES OF SETS
}

\author{
A. N. SHARKOVSKII \\ Institute of Mathematics, Academy of Sciences of the Ukrainian S.S.R. \\ Kiev, U.S.S.R.
}

In the theory of dynamical systems, along with open or closed sets such as basins of sinks, nonwandering sets, centers of dynamical systems, one considers sets with more complicated structure. There appear $F_{\sigma}$ sets, which are unions of not more than countably many closed sets, such as the set of all periodic points, $G_{\delta}$ sets, which are intersections of not more than countably many open sets, such as the set of all orbitally stable points, $F_{\sigma \delta}$ sets, which are intersections of not more than countably many $F_{\sigma}$ sets etc. Sometimes instead of the above Hausdorff classification one uses Baire (Lusin-de la Vallée Poussin) classification. In the latter, open sets and closed sets together with all sets which are both $F_{\sigma}$ and $G_{\delta}$ belong to the first class. The second class consists of sets which are $F_{\sigma}$ sets or $G_{\delta}$ but not both and of sets which are at the same time $F_{\sigma \delta}$ and $G_{\delta \sigma}$ but do not belong to the first class. Further classes are defined in a similar way.

Usually upper descriptive estimates are obtained easily for dynamical systems on an arbitrary space with countable basis of its topology. It turns out that those upper estimates can be reached already on one dimensional systems. Therefore, from the point of view of descriptive set theory, one dimensional dynamical systems can be as complicated as dynamical systems on arbitrary topological spaces ${ }^{(1)}$. Later on we shall give descriptive estimates for the sets most frequently used in the theory of dynamical systems, which are related to such notions as attraction of points, their stability, recurrence.

1. Let $(X, f)$ be a dynamical system with discrete time, let $X$ be a separable

(1) In some sense this statement is no surprise: the real line is so rich that it meets almost all needs of descriptive set theory. On the other hand, if we restrict our attention to those dynamical systems which are group actions (as opposed to semigroups) then the dynamics on the real line is quite simple and it suffices to deal with open or closed sets only. 